\title{
On the Minimal Pseudo-Codewords of Codes from Finite Geometries
}

\author{
Pascal O. Vontobel*, Roxana Smarandache ${ }^{\dagger}$, Negar Kiyavash ${ }^{\ddagger}$, Jason Teutsch ${ }^{\S}$, Dejan Vukobratovic ${ }^{\llbracket}$ \\ * Dept. of ECE, University of Wisconsin, Madison, WI 53706, USA, vontobel@ece.wisc.edu \\ $\dagger$ Dept. of Math. and Stat., San Diego State University, San Diego, CA 92182, USA, rsmarandemath.sdsu.edu \\ $\ddagger$ CSL and Dept. of ECE, University of Illinois, Urbana, IL 61801, USA, kiyavash@uiuc.edu \\ $\S$ Dept. of Math., Indiana University, Bloomington, IN 47405, USA, jteut sch@indiana . edu \\ I Dept. of EE, University of Novi Sad, 21000 Novi Sad, Serbia and Montenegro, de janv@uns.ns .ac.yu
}

\begin{abstract}
In order to understand the performance of a code under maximum-likelihood (ML) decoding, it is crucial to know the minimal codewords. In the context of linear programming (LP) decoding, it turns out to be necessary to know the minimal pseudo-codewords. This paper studies the minimal codewords and minimal pseudo-codewords of some families of codes derived from projective and Euclidean planes. Although our numerical results are only for codes of very modest length, they suggest that these code families exhibit an interesting property. Namely, all minimal pseudo-codewords that are not multiples of a minimal codeword have an AWGNC pseudo-weight that is strictly larger than the minimum Hamming weight of the code. This observation has positive consequences not only for LP decoding but also for iterative decoding.
\end{abstract}

\section{INTRODUCTION}

Our motivation for looking at minimal codewords and minimal pseudo-codewords (PCWs) is twofold. On the one hand we would like to be able to give performance guarantees of the LP decoder, on the other hand, the connection made by Koetter and Vontobel [1], [2] between iterative decoding and LP decoding suggests that results for LP decoding have immediate implications for iterative decoding. In this paper we focus solely on certain families of codes based on projective and Euclidean planes. One of the reasons why these families are worthwhile study objects is that in the past, several groups of authors have experimentally observed that codes from these families can perform very well under iterative decoding, see e.g. [3], [4]. Another reason is that these families of codes have concise descriptions and large automorphism groups which may potentially be used to simplify their analysis.

More precisely, the codes under investigation are the families of codes that were called type-I PG-LDPC and type-I EG-LDPC codes in [4]. Type-I PG-LDPC codes are defined as follows. Let $q \triangleq 2^{s}$ for some positive integer $s$ and consider a (finite) projective plane $\mathrm{PG}(2, q)$ (see e.g. [5]) with $q^{2}+q+1$ points and $q^{2}+q+1$ lines: each point lies on $q+1$ lines and each line contains $q+1$ points. ${ }^{1}$ A standard way of associating a parity-check matrix $\mathbf{H}$ of a binary linear code to a finite geometry is to let the columns of $\mathbf{H}$ correspond to the set of points, to let the rows of $\mathbf{H}$ correspond to the set of lines, and finally to define the entries of $\mathbf{H}$ according to the incidence structure of the finite geometry. In this way, we can associate to the projective plane $\operatorname{PG}(2, q)$ the code $\mathcal{C}_{\mathrm{PG}(2, q)}$ with paritycheck matrix $\mathbf{H} \triangleq \mathbf{H}_{\mathrm{PG}(2, q)}$ whose parameters are:

\footnotetext{
${ }^{1}$ Note that the "2" in $\operatorname{PG}(2, q)$ stands for the dimensionality of the geometry, which in the case of planes is 2 .
}

$\begin{array}{ll}\text { length } & n=q^{2}+q+1 \\ \text { dimension } & k=n-3^{s}-1 \\ \text { minimum Hamming distance } & d_{\text {min }}=q+2 \\ \text { uniform column weight of } \mathbf{H} & w_{\text {col }}=q+1 \\ \text { uniform row weight of } \mathbf{H} & w_{\text {row }}=q+1 \\ \text { size of } \mathbf{H} & n \times n\end{array}$

Type-I EG-LDPC codes are defined as follows. Let $q \triangleq 2^{s}$ for some positive integer $s$ and consider a (finite) Euclidean plane $\operatorname{EG}(2, q)$ (see e.g. [5]) with $q^{2}$ points and $q^{2}+q$ lines: each point lies on $q+1$ lines and each line contains $q$ points. We essentially use the same procedure as outlined above in order to associate a parity-check matrix to a finite geometry. But before doing this, we modify the Euclidean plane slightly: we select a point of $\operatorname{EG}(2, q)$ and remove it together with the $q+1$ lines through it. Doing so, we obtain an $\operatorname{EG}(2, q)$-based code $\mathcal{C}_{\mathrm{EG}(2, q)}$ with parity-check matrix $\mathbf{H} \triangleq \mathbf{H}_{\mathrm{EG}(2, q)}$ whose parameters are:

$\begin{array}{ll}\text { length } & n=q^{2}-1 \\ \text { dimension } & k=n-3^{s}+1 \\ \text { minimum Hamming distance } & d_{\text {min }}=q+1 \\ \text { uniform column weight of } \mathbf{H} & w_{\text {col }}=q \\ \text { uniform row weight of } \mathbf{H} & w_{\text {row }}=q \\ \text { size of } \mathbf{H} & n \times n\end{array}$

Both families of codes have the nice property that with an appropriate ordering of the columns and rows, the paritycheck matrix is a circulant matrix, meaning that $\mathcal{C}_{\mathrm{PG}(2, q)}$ and $\mathcal{C}_{\mathrm{EG}(2, q)}$ are cyclic codes. This fact can e.g. be used for efficient encoding. Such symmetries can also substantially simplify the analysis; let us point out that the automorphism groups of $\mathcal{C}_{\mathrm{PG}(2, q)}$ and $\mathcal{C}_{\mathrm{EG}(2, q)}$ actually contain many more automorphisms besides the cyclic-shift automorphism implied by the cyclicity of the codes.

The structure of the rest of the paper is as follows. Sec. III discusses ML and LP decoding and Secs. III and IV introduce minimal codewords and minimal PCWs, respectively. The aim of these earlier sections is to set the stage for Sec. $\nabla$ and to enable the reader to appreciate the numerical results presented therein for certain selected codes. Finally, in Sec. VI] we state some concluding remarks.

\section{ML AND LP DECODING}

In this section we briefly review ML and LP decoding. Consider a binary linear code $\mathcal{C}$ of length $n$ and dimension $k$ 
that is used for data transmission over a memoryless binaryinput channel. The codeword that is transmitted will be called $\mathbf{x}$ whereas the received vector will be called $\mathbf{y}$. Based on the received vector, we can define the log-likelihood ratios (LLRs) to be $\lambda_{i} \triangleq \log \left(p_{Y_{i} \mid X_{i}}\left(y_{i} \mid 0\right) / p_{Y_{i} \mid X_{i}}\left(y_{i} \mid 1\right)\right), i=1, \ldots, n$. ML decoding can then be cast as

$$
\hat{\mathbf{x}} \triangleq \arg \min _{\mathbf{x} \in \mathcal{C}} \sum_{i=1}^{n} x_{i} \lambda_{i},
$$

Letting $\operatorname{conv}(\mathcal{C})$ be the convex hull of $\mathcal{C}$ in $\mathbb{R}^{n}$, the above ML decoding rule can also be formulated as

$$
\hat{\mathbf{x}} \triangleq \arg \min _{\mathbf{x} \in \operatorname{conv}(\mathcal{C})} \sum_{i=1}^{n} x_{i} \lambda_{i} .
$$

Unfortunately, for most codes of interest, the description complexity of $\operatorname{conv}(\mathcal{C})$ grows exponentially in the block length and therefore finding the minimum in (2) with a linear programming solver is highly impractical for reasonably long codes. $^{2}$

The next step is to use a standard approach from optimization practice: we replace the minimization over $\operatorname{conv}(\mathcal{C})$ by a minimization over some easily describable polytope $\mathcal{P}$ that is a relaxation of $\operatorname{conv}(\mathcal{C})$ :

$$
\hat{\mathbf{x}} \triangleq \arg \min _{\mathbf{x} \in \mathcal{P}} \sum_{i=1}^{n} x_{i} \lambda_{i} .
$$

If $\mathcal{P}$ is strictly larger than $\operatorname{conv}(\mathcal{C})$ then the decision rule in (3) obviously represents a sub-optimal decoder. A relaxation that works particularly well for LDPC codes is given by the following approach [6], [7]. Let $\mathcal{C}$ be described by an $m \times n$ parity-check matrix $\mathbf{H}$ with rows $\mathbf{h}_{1}, \mathbf{h}_{2}, \ldots, \mathbf{h}_{m}$. Then the polytope $\mathcal{P} \triangleq \mathcal{P}(\mathbf{H})$, in this context also called the fundamental polytope [1], is defined as

$$
\mathcal{P} \triangleq \bigcap_{i=1}^{m} \operatorname{conv}\left(\mathcal{C}_{i}\right) \text { with } \mathcal{C}_{i} \triangleq\left\{\mathbf{x} \in\{0,1\}^{n} \mid \mathbf{h}_{i} \mathbf{x}^{\top}=0 \bmod 2\right\} .
$$

Note that $\mathcal{P}$ is a convex set within $[0,1]^{n}$ that contains $\operatorname{conv}(\mathcal{C})$ but whose description complexity is much smaller than the one of $\operatorname{conv}(\mathcal{C})$. Points in the set $\mathcal{P}$ will be called PCWs. Because the set $\mathcal{P}$ is usually strictly larger than $\operatorname{conv}(\mathcal{C})$, it can obviously happen that the decoding rule in (3) delivers a vertex of $\mathcal{P}$ that is not a codeword. Such vertices are the reason for the sub-optimality of LP decoding (cf. [1], [7]). Note that $\mathcal{P}(\mathbf{H})$ is a function of the parity-check matrix $\mathbf{H}$ that describes the code $\mathcal{C}$; different parity-check matrices for the same code might therefore lead to different fundamental polytopes.

\section{Minimal Codewords}

Although ML decoding is often impractical, knowing bounds on the block error rate of an ML decoder can help in assessing the performance of sub-optimal but practical decoding algorithms.

Definition 1 (cf. e.g. [8], [9]): Let $\mathcal{C}$ be a binary linear code of length $n$. For $\mathbf{x} \in \mathcal{C}$, let $\mathcal{D}_{\mathbf{x}}^{\mathrm{ML}} \triangleq\left\{\boldsymbol{\lambda} \in \mathbb{R}^{n} \mid \mathbf{x}^{\prime} \cdot \boldsymbol{\lambda}^{\top} \geq\right.$

\footnotetext{
${ }^{2}$ Exceptions to this observation include for example the class of convolutional codes with not too many states.
}

$\mathbf{x} \cdot \boldsymbol{\lambda}^{\top}$ for all $\left.\mathbf{x}^{\prime} \in \mathcal{C} \backslash\{\mathbf{x}\}\right\}$ be the region in the LLR space where the ML decoder decides in favor of the codeword $x .^{3}$

In the following, we will assume that we use a binary linear code $\mathcal{C}$ for data transmission over a binary-input outputsymmetric channel. For this setup, when studying the ML decoder in (1) or (2), we can without loss of generality assume that the zero codeword was sent, because all decision regions are congruent.

Our interest in the following definition will become apparent in Th. 3 below.

Definition 2: Let the support of a vector $\mathbf{x}$ be defined as $\operatorname{supp}(\mathbf{x}) \triangleq\left\{i \mid x_{i} \neq 0\right\}$ and let $\mathcal{C}$ be a binary code. A non-zero codeword $\mathbf{x} \in \mathcal{C}$ is called minimal if and only if its support does not (strictly) contain the support of any other non-zero codeword as a proper subset. The set of all minimal codewords of $\mathcal{C}$ will be denoted by $\mathcal{M}(\mathcal{C})$.

Theorem 3 (cf. e.g. [9]): Let $\mathcal{C}$ be a binary linear code of length $n$. The decision region $\mathcal{D}_{\mathbf{x}}^{\mathrm{ML}}$ of a codeword $\mathbf{x} \in \mathcal{C}$ shares a facet with the decision region $\mathcal{D}_{\mathbf{0}}^{\mathrm{ML}}$ of the zero codeword if and only if $\mathbf{x} \in \mathcal{M}(\mathcal{C})$.

Therefore, knowing the minimal codewords of the code $\mathcal{C}$ is sufficient in order to assess its ML decoding performance. (Further results about minimal codewords can e.g. be found in [8]-[11].)

\section{The Fundamental CONE AND MINIMAL PSEUdO-CODEWORDS}

For LP decoding of a binary linear code that is used for data transmission over a binary-input output-symmetric channel, it is sufficient to consider the part of the fundamental polytope $\mathcal{P}$ around the vertex 0, cf. [1]. (See also [6], [7] that discuss this so-called " $\mathcal{C}$-symmetry" property.)

Definition 4 ([1], [7]): Let $\mathcal{C}$ be an arbitrary binary linear code and let $\mathbf{H}$ be its parity-check matrix. We let $\mathcal{J} \triangleq \mathcal{J}(\mathbf{H})$ be the set of row indices of $\mathbf{H}$ and we let $\mathcal{I} \triangleq \mathcal{I}(\mathbf{H})$ be the set of column indices of $\mathbf{H}$, respectively. For each $j \in \mathcal{J}$, we let $\mathcal{I}_{j} \triangleq \mathcal{I}_{j}(\mathbf{H}) \triangleq\left\{i \in \mathcal{I} \mid h_{j i}=1\right\}$. We define the fundamental cone $\mathcal{K}(\mathbf{H})$ of $\mathbf{H}$ to be the set of vectors $\omega \in \mathbb{R}^{n}$ that satisfy

$$
\begin{aligned}
& \forall i \in \mathcal{I}: \omega_{i} \geq 0, \\
& \forall j \in \mathcal{J}, \forall i \in \mathcal{I}_{j}: \quad \sum_{i^{\prime} \in \mathcal{I}_{j} \backslash\{i\}} \omega_{i^{\prime}} \geq \omega_{i} .
\end{aligned}
$$

The fundamental cone defined in Def. 4 is exactly the part of the fundamental polytope $\mathcal{P}$ around the vertex $\mathbf{0}$ and stretched to infinity. We note that if $\boldsymbol{\omega} \in \mathcal{K}(\mathbf{H})$, then also $\alpha \cdot \boldsymbol{\omega} \in \mathcal{K}(\mathbf{H})$ for any $\alpha>0$. Moreover, for any $\boldsymbol{\omega} \in \mathcal{K}(\mathbf{H})$ there exists an $\alpha>0$ (in fact, a whole interval of $\alpha$ 's) such that $\alpha \cdot \boldsymbol{\omega} \in \mathcal{P}(\mathbf{H})$.

For a given binary linear $\operatorname{code} \mathcal{C}$ with parity-check matrix $\mathbf{H}$, the importance of the set $\mathcal{K}(\mathbf{H})$ lies in the following fact. Let $\mathcal{D}_{\mathbf{0}}^{\mathrm{LP}} \triangleq\left\{\boldsymbol{\lambda} \in \mathbb{R}^{n} \mid \boldsymbol{\omega} \cdot \boldsymbol{\lambda}^{\top} \geq 0\right.$ for all $\left.\boldsymbol{\omega} \in \mathcal{P}(\mathbf{H}) \backslash\{\mathbf{0}\}\right\}$ be the region where the LP decoder decides in favor of the codeword 0. ${ }^{4}$ It can easily be seen that $\mathcal{D}_{\mathbf{0}}^{\mathrm{LP}}=\left\{\boldsymbol{\lambda} \in \mathbb{R}^{n} \mid \boldsymbol{\omega} \cdot \boldsymbol{\lambda}^{\top} \geq\right.$ 0 for all $\boldsymbol{\omega} \in \mathcal{K}(\mathbf{H}) \backslash\{\mathbf{0}\}\}$. Therefore, when studying LP

\footnotetext{
${ }^{3}$ Note that during ML decoding, ties between decoding regions can either be resolved in a random or in a systematic fashion.

${ }^{4}$ Note that during LP decoding, ties between decoding regions can either be resolved in a random or in a systematic fashion.
} 
decoding it is enough to know $\mathcal{K}(\mathbf{H})$; all vectors $\boldsymbol{\omega} \in \mathcal{K}(\mathbf{H})$ will henceforth be called PCWs.

Definition 5 ([1]): Let $\mathcal{C}$ be an arbitrary binary linear code described by the parity-check matrix $\mathbf{H}$ whose fundamental cone is $\mathcal{K}(\mathbf{H})$. A vector $\boldsymbol{\omega} \in \mathcal{K}(\mathbf{H})$ is called a minimal $P C W$ if the set $\{\alpha \cdot \boldsymbol{\omega} \mid \alpha \geq 0\}$ is an edge of $\mathcal{K}(\mathbf{H})$. Moreover, the set of all minimal PCWs will be called $\mathcal{M}_{\mathrm{p}}(\mathcal{K}(\mathbf{H})){ }^{5}$

For a given binary linear code $\mathcal{C}$ with parity-check matrix $\mathbf{H}$, the importance of the set $\mathcal{M}_{\mathrm{p}}(\mathcal{K}(\mathbf{H}))$ lies in the following fact. From basic cone properties (cf. e.g. [12]), it can easily be seen that $\mathcal{D}_{\mathbf{0}}^{\mathrm{LP}}=\left\{\boldsymbol{\lambda} \in \mathbb{R}^{n} \mid \boldsymbol{\omega} \cdot \boldsymbol{\lambda}^{\top} \geq 0\right.$ for all $\left.\boldsymbol{\omega} \in \mathcal{M}_{\mathrm{p}}(\mathcal{K}(\mathbf{H}))\right\}$. Therefore, the set $\mathcal{M}_{\mathrm{p}}(\mathcal{K}(\mathbf{H}))$ completely characterizes the behavior of the LP decoder.

Definition 6: Let $\mathcal{C}$ be an arbitrary binary linear code described by the parity-check matrix $\mathbf{H}$. The additive white Gaussian noise channel (AWGNC) pseudo-weight [13] of a PCW $\boldsymbol{\omega} \in \mathcal{K}(\mathbf{H})$ is defined to be $w_{\mathrm{p}}^{\mathrm{AWGNC}}(\boldsymbol{\omega})=$ $\|\boldsymbol{\omega}\|_{1}^{2} /\|\boldsymbol{\omega}\|_{2}^{2}$, where $\|\boldsymbol{\omega}\|_{1}$ and $\|\boldsymbol{\omega}\|_{2}$ are the $L_{1^{-}}$and $L_{2^{-}}$ norm of $\omega$, respectively. ${ }^{6}$

The significance of $w_{\mathrm{p}}^{\mathrm{AWGNC}}(\boldsymbol{\omega})$ is the following: it can be shown that the squared Euclidean distance from the point $+\mathbf{1}$ in signal space (which corresponds to the codeword $\mathbf{0}$ ) to the plane $\left\{\boldsymbol{\lambda} \in \mathbb{R}^{n} \mid \boldsymbol{\omega} \cdot \boldsymbol{\lambda}^{\top}=0\right\}$ is $w_{\mathrm{p}}^{\mathrm{AWGNC}}(\boldsymbol{\omega})$.

Definition 7: Let $\mathcal{C}$ be an arbitrary binary linear code. We define the codeword weight enumerator and the minimal codeword weight enumerator to be the polynomials

$$
\chi_{\mathcal{C}}^{\mathrm{CW}}(X) \triangleq \sum_{\mathbf{x} \in \mathcal{C}} X^{w_{\mathrm{H}}(\mathbf{x})} \quad \text { and } \quad \chi_{\mathcal{C}}^{\mathrm{MCW}}(X) \triangleq \sum_{\mathbf{x} \in \mathcal{M}(\mathcal{C})} X^{w_{\mathrm{H}}(\mathbf{x})},
$$

respectively.

Definition 8: Let $\mathcal{C}$ be an arbitrary binary linear code described by the parity-check matrix $\mathbf{H}$. We define the minimal $P C W A W G N C$ pseudo-weight enumerator to be the polynomial (with potentially non-integer exponents)

$$
\chi_{\mathbf{H}}^{\mathrm{MPCW}, \operatorname{AWGNC}}(X)=\sum_{[\boldsymbol{\omega}] \in \mathcal{M}_{\mathrm{p}}(\mathcal{K}(\mathbf{H}))} X^{w_{\mathrm{p}}^{\mathrm{AWGNC}}(\boldsymbol{\omega})},
$$

where the summation is over all equivalence classes of minimal PCWs. ${ }^{7}$

Definition 9: Let $\mathcal{C}$ be an arbitrary binary linear code described by the parity-check matrix $\mathbf{H}$ and let $\mathcal{M}_{\mathrm{p}}^{\prime}(\mathcal{K}(\mathbf{H}))$ be the set of all minimal PCWs that are not multiples of minimal codewords. We call the real-valued quantity

$$
g(\mathbf{H}) \triangleq \min _{\boldsymbol{\omega} \in \mathcal{M}_{\mathrm{p}}^{\prime}(\mathcal{K}(\mathbf{H}))} w_{\mathrm{p}}^{\mathrm{AWGNC}}(\boldsymbol{\omega})-w_{\mathrm{H}}^{\min }(\mathcal{C}(\mathbf{H}))
$$

the pseudo-weight spectrum gap of $\mathbf{H}$.

Using Cor. 8 in [1] one can show that for a randomly constructed $\left(w_{\text {col }}, w_{\text {row }}\right)$-regular code with $3 \leq w_{\text {col }}<w_{\text {row }}$ the pseudo-weight spectrum gap becomes strictly negative with probability one as the block length goes to infinity. However, using Th. 1 in [14] one can show that for the PG $(2, q)$ - and $\mathrm{EG}(2, q)$-based codes (with square parity-check matrix as discussed in Sec. I) the pseudo-weight spectrum gap is non-negative. In fact, we will see that for the codes

\footnotetext{
${ }^{5}$ Note that this definition implies that $\mathbf{0} \notin \mathcal{M}_{\mathrm{p}}(\mathcal{K}(\mathbf{H}))$.

${ }^{6}$ We set $w_{\mathrm{p}}^{\mathrm{AWGNC}}(\mathbf{x}) \triangleq 0$ for $\mathbf{x}=\mathbf{0}$. Note that for $\mathbf{x} \in\{0,1\}^{n}$ we have $w_{\mathrm{p}}^{\mathrm{AWGNC}}(\mathbf{x})=w_{\mathrm{H}}(\mathbf{x})$, where $w_{\mathrm{H}}(\mathbf{x})$ is the Hamming weight of $\mathbf{x}$.

${ }^{7}$ Two PCWs $\boldsymbol{\omega}, \boldsymbol{\omega}^{\prime} \in \mathcal{K}(\mathbf{H})$ are in the same equivalence class if there exists an $\alpha>0$ such that $\boldsymbol{\omega}=\alpha \cdot \boldsymbol{\omega}^{\prime}$.
}

investigated in Sec. V the pseudo-weight spectrum gap is significantly positive. We note that by applying simple performance bounding techniques it can be shown that the larger the gap is, the closer is the LP decoding performance (and potentially also the iterative decoding performance [1]) to the ML decoding performance as the SNR goes to infinity.

Before we turn to some numerical results about minimal codewords and minimal PCWs, let us mention some related work by Kashyap and Vardy [15] which discusses results that characterize (minimal) stopping sets for finite-geometry-based codes. This is of some relevance to this paper because it is well-known that the support set of any PCW is a stopping set and that for any stopping set there exists a PCW whose support set equals that stopping set [1], [7].

\section{NumericAl RESUlts}

In this section we present minimal PCWs, weight enumerators, and the pseudo-weight spectrum gap for some short $\operatorname{PG}(2, q)$ - and $\operatorname{EG}(2, q)$-based codes.

\section{A. Type-I PG-LDPC code for $q=2$}

The $\mathrm{PG}(2,2)$-based code $\mathcal{C}_{\mathrm{PG}(2,2)}$ of type I has parameters $\left[n=7, k=3, d_{\min }=4\right]$ and can be represented by the following circulant parity-check matrix of size $7 \times 7$ :

$$
\mathbf{H}_{\mathrm{PG}(2,2)}=\left(\begin{array}{ccccccc}
1 & 1 & 0 & 1 & 0 & 0 & 0 \\
0 & 1 & 1 & 0 & 1 & 0 & 0 \\
0 & 0 & 1 & 1 & 0 & 1 & 0 \\
0 & 0 & 0 & 1 & 1 & 0 & 1 \\
1 & 0 & 0 & 0 & 1 & 1 & 0 \\
0 & 1 & 0 & 0 & 0 & 1 & 1 \\
1 & 0 & 1 & 0 & 0 & 0 & 1
\end{array}\right) .
$$

The set $\mathcal{M}(\mathcal{C})$ of minimal codewords consists of the following codewords:

$(1,0,0,1,0,1,1),(1,1,0,0,1,0,1),(1,1,1,0,0,1,0),(0,1,1,1,0,0,1)$, $(1,0,1,1,1,0,0),(0,1,0,1,1,1,0),(0,0,1,0,1,1,1)$.

Obviously, all of them have Hamming weight 4 and they are all cyclic shifts of each other. Because the code has $2^{3}=8$ codewords in total, it turns that this code is special in the following sense: there are no non-zero codewords that are not minimal codewords.

The set $\mathcal{M}_{\mathrm{p}}(\mathcal{K}(\mathbf{H}))$ of minimal codewords contains all the elements from $\mathcal{M}(\mathcal{C})$ plus the following PCWs that are not codewords (we show one representative per equivalence class):

$(1,2,2,1,2,1,1),(1,1,2,2,1,2,1),(1,1,1,2,2,1,2),(2,1,1,1,2,2,1)$, $(1,2,1,1,1,2,2),(2,1,2,1,1,1,2),(2,2,1,2,1,1,1)$.

All these minimal PCWs that are not codewords turn out to be cyclic shifts of each other and to have AWGNC pseudoweight $\frac{100}{16}=6.25$. The weight enumerators for this code are therefore:

$$
\begin{aligned}
& \chi_{\mathcal{C}_{\mathrm{PG}(2,2)}^{\mathrm{CW}}}^{\mathrm{C}}(X)=X^{0}+7 X^{4}, \\
& \chi_{\mathcal{C}_{\mathrm{PG}(2,2)}^{\mathrm{MCW}}}^{\mathrm{M}}(X)=7 X^{4},
\end{aligned}
$$

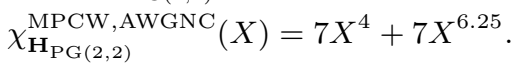

Hence, the pseudo-weight spectrum gap is $g\left(\mathbf{H}_{\mathrm{PG}(2,2)}\right)=$ $6.25-4=2.25$.

The codes introduced in Sec. Iwere based on square paritycheck matrices. However, the code $\operatorname{PG}(2,2)$ can also be described by a parity-check matrix $\mathbf{H}_{\mathrm{PG}(2,2)}^{\prime}$ of size $4 \times 7$ 


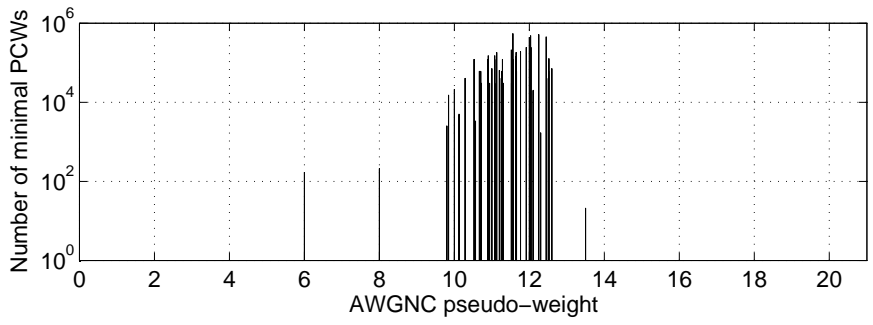

Fig. 1. Histogram of the AWGNC pseudo-weight of minimal PCWs of the $\mathrm{PG}(2,4)$-based code. (Note that the $\mathrm{y}$-axis is logarithmic.)

which is equivalent to the first four lines of the $\mathbf{H}_{\mathrm{PG}(2,2)}$. The minimal PCWs that are not codewords turn out to be (we show one representative per equivalence class):

$(3,2,1,1,1,0,0),(0,1,2,1,1,3,0),(0,1,1,1,2,0,3),(0,1,1,1,1,0,0)$, $(2,1,1,1,0,0,1),(2,1,0,1,1,1,0),(1,2,1,1,1,0,0),(0,1,2,1,1,1,0)$, $(0,1,1,1,2,0,1),(0,1,1,1,0,2,1),(1,0,1,1,1,2,0),(1,0,1,1,1,0,2)$, $(0,1,0,1,1,1,2)$,

from which follows that

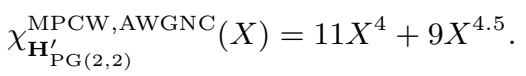

Note that the pseudo-weight spectrum gap is $g\left(\mathbf{H}_{\mathrm{PG}(2,2)}^{\prime}\right)=$

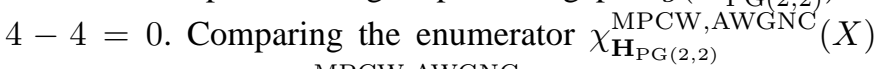

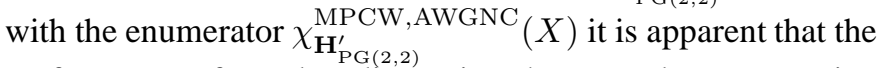
performance of LP decoding using the second representation will be worse than the performance of LP decoding using the first representation. Based on iterative decoder simulations, MacKay and Davey [16, Sec. 4] observed a similar performance hierarchy between different representations of the same code. (Note that the code under investigation in [16] was the PG(2,16)-based code.)

\section{B. Type-I PG-LDPC code for $q=4$}

The parity-check matrix $\mathbf{H}_{\mathrm{PG}(2,4)}$ of the $\mathrm{PG}(2,4)$-based code $\mathcal{C}_{\mathrm{PG}(2,4)}$ has size $21 \times 21$, uniform column and row weight 5 , and yields a code with parameters $\left[n=21, k=11, d_{\min }=6\right]$. The codeword weight enumerator and the minimal codeword weight enumerator are

$$
\begin{aligned}
\chi_{\mathcal{C}_{\mathrm{PG}(2,4)}^{\mathrm{CW}}}^{\mathrm{CW}=} & X^{0}+168 X^{6}+210 X^{8}+1008 X^{10} \\
& +280 X^{12}+360 X^{14}+21 X^{16} \\
\chi_{\mathcal{C}_{\mathrm{PG}(2,4)}^{\mathrm{MCW}}}^{\mathrm{MC}}= & 168 X^{6}+210 X^{8}+1008 X^{10}
\end{aligned}
$$

respectively. Looking at these enumerators we see that all codewords with Hamming weight 6,8 , and 10 are also minimal codewords. Analyzing the set of all weight 6 codewords one sees that they all have the same pattern, i.e. they can all be obtained from a single weight- 6 codeword by applying a suitable $\mathrm{PG}(2,4)$-automorphism. The same is true for all other sets of codewords with the same weight. This makes the classification of all the codewords of $\mathcal{C}_{\mathrm{PG}(2,4)}$, and in particular of the minimal codewords of $\mathcal{C}_{\mathrm{PG}(2,4)}$, relatively easy.

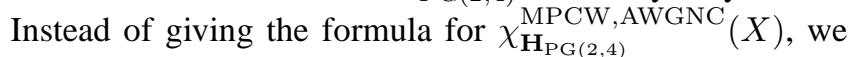
simply give its histogram (spectrum), cf. Fig. 1 The gap turns out to be $g\left(\mathbf{H}_{\mathrm{PG}(2,4)}\right)=9.8-6=3.8$.
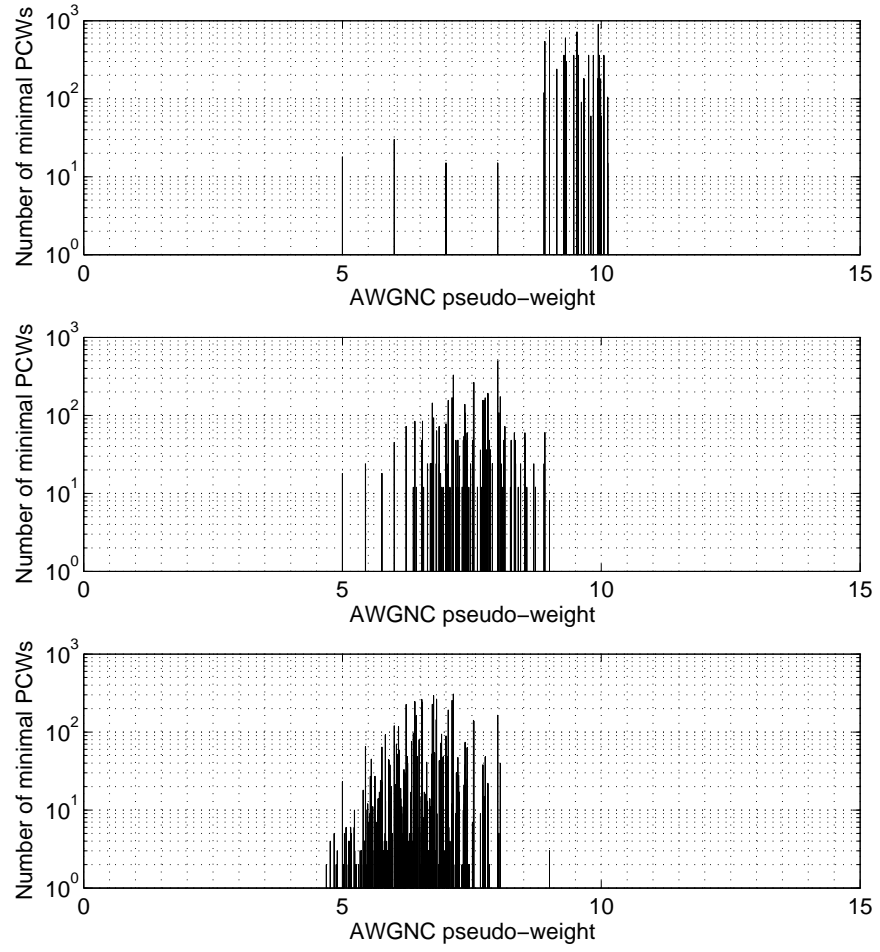

Fig. 2. Histogram of the AWGNC pseudo-weight of minimal PCWs of the $\mathrm{EG}(2,4)$-based code. (Note that the $\mathrm{y}$-axis is logarithmic.) Top: For $15 \times 15$ parity-check matrix $\mathbf{H}_{\mathrm{EG}(2,4)}, g\left(\mathbf{H}_{\mathrm{EG}(2,4)}\right)=\frac{169}{19}-5 \approx 8.89-5=3.89$. Middle: For $9 \times 15$ parity-check matrix $\mathbf{H}_{\mathrm{EG}(2,4)}^{\prime}, g\left(\mathbf{H}_{\mathrm{EG}(2,4)}^{\prime}\right)=\frac{49}{9}-$ $5 \approx 5.44-5=0.44$. Bottom: For $8 \times 15$ parity-check matrix $\mathbf{H}_{\mathrm{EG}(2,4)}^{\prime \prime}$, $g\left(\mathbf{H}_{\mathrm{EG}(2,4)}^{\prime \prime}\right)=\frac{361}{77}-5 \approx 4.69-5=-0.31$.

\section{Type-I PG-LDPC code for $q=8$}

Judging from some very preliminary results (based on random search experiments) the pseudo-weight spectrum gap $g\left(\mathbf{H}_{\mathrm{PG}(2,8)}\right)$ for the $\mathrm{PG}(2,8)$-based code seems to be at least 6.0 .

\section{Type-I EG-LDPC code for $q=4$}

The parity-check matrix $\mathbf{H}_{\mathrm{EG}(2,4)}$ of the $\mathrm{EG}(2,4)$-based code $\mathcal{C}_{\mathrm{EG}(2,4)}$ has size $15 \times 15$, uniform column and row weight 4 , and yields a code with parameters $\left[n=15, k=7, d_{\min }=5\right]$. The codeword weight enumerator and the minimal codeword weight enumerator are

$$
\begin{aligned}
& \chi_{\mathcal{C}_{\mathrm{EG}(2,4)}^{\mathrm{CW}}}^{\mathrm{CW}}= X^{0}+18 X^{5}+30 X^{6}+15 X^{7} \\
&+15 X^{8}+30 X^{9}+18 X^{10}+X^{15} \\
& \chi_{\mathcal{C}_{\mathrm{EG}(2,4)}^{\mathrm{MCW}}(X)=} 18 X^{5}+30 X^{6}+15 X^{7}+15 X^{8}+30 X^{9},
\end{aligned}
$$

respectively. Looking at these enumerators we see that all codewords with Hamming weight 5, 6, 7, 8, and 9 are also minimal codewords. Analyzing the set of all weight5 codewords one sees that they all have the same pattern, i.e. they can all be obtained from a single weight- 5 codeword by applying a suitable $\operatorname{EG}(2,4)$-automorphism. The same is true for all other sets of codewords with the same weight.

The histograms (spectra) in Fig. 2 correspond to various parity-check matrices that describe $\mathcal{C}_{\mathrm{EG}(2,4)}$. Fig. 2] (top) 


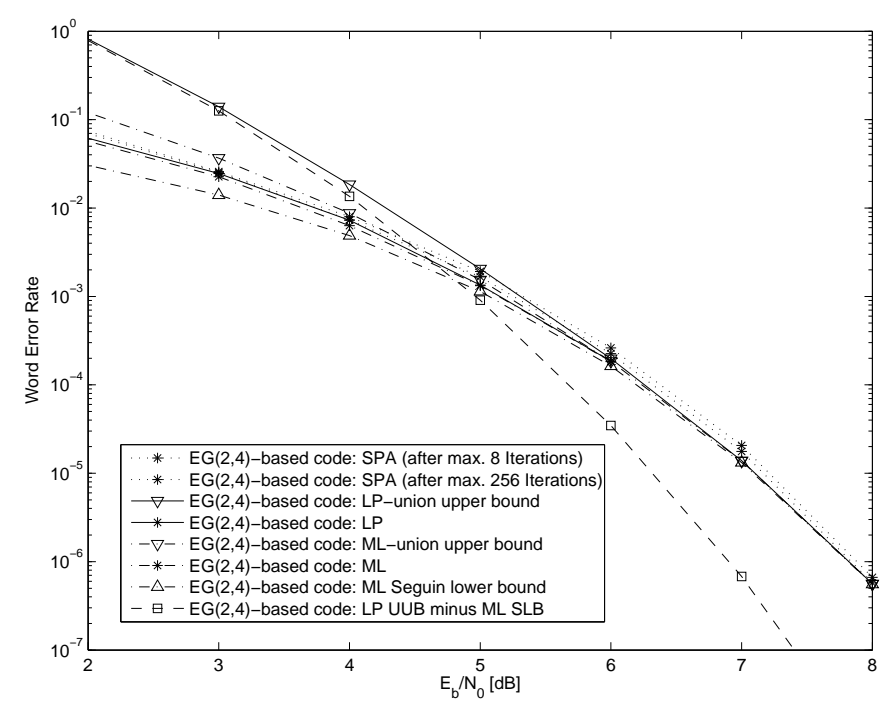

Fig. 3. Word error rate for various decoding algorithms together with some upper and lower bounds. (See main text for explanations.)

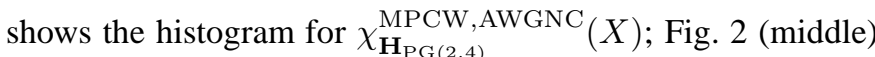
shows the histogram for $\chi_{\mathbf{H}_{\mathrm{PG}(2,4)}^{\prime}}^{\mathrm{MPCW}, \mathrm{AWGNC}}(X)$ where $\mathbf{H}_{\mathrm{PG}(2,4)}^{\prime}$ is a randomly selected $9 \times 15$ submatrix (with column weights at least 2) of $\mathbf{H}_{\mathrm{PG}(2,4)}$; and finally Fig. 2 (bottom) shows the histogram for $\chi_{\mathbf{H}_{\mathrm{PG}(2,4)}^{\mathrm{MPCW}} \text {, AWGNC }}^{\prime \prime}(X)$ where $\mathbf{H}_{\mathrm{PG}(2,4)}^{\prime \prime}$ is an $8 \times 15$ submatrix (with five columns having weight only one) of consecutive rows of the (circulant) matrix $\mathbf{H}_{\mathrm{PG}(2,4)}$. It can easily be seen that for the parity-check matrices under investigation those with more lines lead to more favorable histograms.

In Fig. 3 we show various decoding simulation results for data transmission over a binary-input AWGNC and lower and upper bounds: $\mathbf{H}_{\mathrm{EG}(2,4)}$-based sum-product algorithm decoding, $\mathbf{H}_{\mathrm{EG}(2,4)}$-based LP decoding, $\mathcal{C}_{\mathrm{EG}(2,4)}$-based $\mathrm{ML}$ decoding, an upper bound on LP decoding based on a union of events upper bound, an upper bound on ML decoding based on a union of events upper bound, and a lower bound on ML decoding based on an inequality by de Caen as presented by Séguin [17]. It can be seen that thanks to the knowledge of minimal codewords and minimal PCWs we are able to obtain bounds that are very tight from a certain SNR value on.

\section{E. How the results were obtained}

Let us briefly mention how the results for the minimal PCWs were obtained. We used the program "lrs" [18] to search edges in cones. For the code $\mathcal{C}_{\mathrm{PG}(2,4)}$ we additionally used the two-transitivity of the points of a projective plane in order to formulate a simpler edge-enumeration subproblem which can be solved efficiently and from which all the minimal PCWs can be derived. There are various other ways to use the large automorphism groups of these codes that help in simplifying the edge-enumerating problem. Properties of minimal codewords might also be used towards that goal.

\section{CONCLUding REMARKS}

We have investigated the minimal PCWs of some simple PG $(2, q)$ - and $\mathrm{EG}(2, q)$-based binary linear codes and we have introduced the notion of a pseudo-weight spectrum gap for a parity-check matrix, a concept which is certainly worthwhile to be further explored. Although our numerical results are for codes of very modest size, to the best of our knowledge this is the first study that tries to analytically quantify the behavior of PG $(2, q)$ - and EG $(2, q)$-based binary linear codes under LP and iterative decoding. Extending these results to somewhat longer codes has the potential to explain many experimental observations made in the past.

\section{ACKNOWLEDGMENT}

This paper is based on work started at the IMA Participating Institutions Summer Program for graduate students on "Coding and Cryptography" at Notre Dame University, Notre Dame, IN, USA, June 7-25, 2004. We would like to take this opportunity to thank the organizers. P.O.V.'s research was supported by NSF Grants CCR 99-84515, CCR 01-05719, and ATM-0296033 and by DOE SciDAC and ONR Grant N0001400-1-0966.

\section{REFERENCES}

[1] R. Koetter and P. O. Vontobel, "Graph covers and iterative decoding of finitelength codes," in Proc. 3rd Intern. Conf. on Turbo Codes and Related Topics, (Brest, France), pp. 75-82, Sept. 1-5 2003.

[2] P. O. Vontobel and R. Koetter, "On the relationship between linear programming decoding and min-sum algorithm decoding," in Proc. Intern. Symp. on Inform. Theory and its Applications (ISITA), (Parma, Italy), pp. 991-996, Oct. 10-13 2004

[3] R. Lucas, M. Fossorier, Y. Kou, and S. Lin, "Iterative decoding of one-step majority logic decodable codes based on belief propagation," IEEE Trans. on Comm. vol. COMM-48, pp. 931-937, June 2000.

[4] Y. Kou, S. Lin, and M. P. C. Fossorier, "Low-density parity-check codes based on finite geometries: a rediscovery and new results," IEEE Trans. on Inform. Theory, vol. IT-47, pp. 2711-2736, Nov. 2001.

[5] L. M. Batten, Combinatorics of Finite Geometries. Cambridge: Cambridge University Press, second ed., 1997.

[6] J. Feldman, Decoding Error-Correcting Codes via Linear Programming. $\mathrm{PhD}$ thesis, Massachusetts Institute of Technology, Cambridge, MA, 2003. Available online under http://www. columbia.edu/ jf2189/pubs.html.

[7] J. Feldman, M. J. Wainwright, and D. R. Karger, "Using linear programming to decode binary linear codes," IEEE Trans. on Inform. Theory, vol. IT-51, no. 3, pp. 954-972, 2005.

[8] T. Y. Hwang, "Decoding linear block codes for minimizing word error rate," IEEE Trans. on Inform. Theory, vol. 25, no. 6, pp. 733-737, 1979.

[9] E. Agrell, "Voronoĭ regions for binary linear block codes," IEEE Trans. on Inform Theory, vol. 42, no. 1, pp. 310-316, 1996

[10] A. Ashikhmin and A. Barg, "Minimal vectors in linear codes," IEEE Trans. on Inform. Theory, vol. 44, no. 5, pp. 2010-2017, 1998.

[11] Y. Borissov, N. Manev, and S. Nikova, "On the non-minimal codewords in the binary Reed-Muller code," in Proc. IEEE Intern. Symp. on Inform. Theory, (Washington, D.C., USA), p. 39, June 24-29 2001.

[12] S. Boyd and L. Vandenberghe, Convex Optimization. Cambridge, UK: Cambridge University Press, 2004.

[13] G. D. Forney, Jr., R. Koetter, F. R. Kschischang, and A. Reznik, "On the effective weights of pseudocodewords for codes defined on graphs with cycles," in Codes, Systems, and Graphical Models (Minneapolis, MN, 1999) (B. Marcus and J. Rosenthal, eds.), vol. 123 of IMA Vol. Math. Appl., pp. 101-112, Springe Verlag, New York, Inc., 2001.

[14] P. O. Vontobel and R. Koetter, "Lower bounds on the minimum pseudo-weigh of linear codes," in Proc. IEEE Intern. Symp. on Inform. Theory, (Chicago, IL, USA), p. 70, June 27-July 22004

[15] N. Kashyap and A. Vardy, "Stopping sets in codes from designs," in Proc. IEEE Intern. Symp. on Inform. Theory, (Pacifico Yokohama, Japan), p. 122, June $29-$ July 42003.

[16] D. J. C. MacKay and M. C. Davey, "Evaluation of Gallager codes for short block length and high rate applications," in Codes, Systems, and Graphical Models (Minneapolis, MN, 1999) (B. Marcus and J. Rosenthal, eds.), pp. 113130, Springer Verlag, New York, Inc., 2001.

[17] G. E. Séguin, "A lower bound on the error probability for signals in white Gaussian noise," IEEE Trans. on Inform. Theory, vol. 44, no. 7, pp. 3168-3175, 1998.

[18] D. Avis, "lrs: A revised implementation of the reverse search vertex enumeration algorithm," in Polytopes - Combinatorics and Computation (G. Kalai and G. M. Ziegler, eds.), pp. 177-198, Birkhäuser-Verlag, 2000. Programs are available online under http://cgm.cs.mcgill.ca/〜avis/c/lrs.html. 